\title{
Thermal Conductivity of Helium
}

Douglas Clark

$8 / 26 / 92$

DØ Engineering Note 3740.510-EN-334

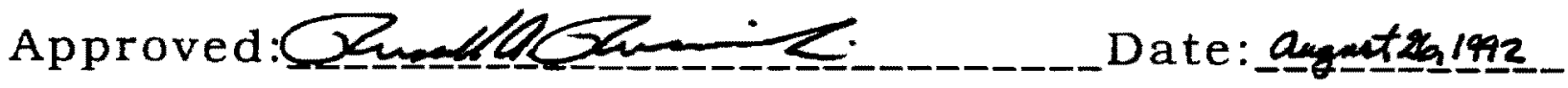


EN-334

$8 / 26 / 92$

\section{Introduction}

This engineering note examines the thermal conductivity of helium as a function of temperature and pressure. The note attempts to determine the effect of varying pressure on the thermal conductivity of the vapor state over various temperature ranges.

\section{Data/Results}

In each of the following figures; 0.01MPa, 0.1MPa, 0.5MPa, and 1.OMPa isobars are plotted versus temperature. Figure 1 shows the thermal conductivity of helium over the temperature range 0-600 K. Figure 2 covers the temperature range $0-300 \mathrm{~K}$. Figure 3 limits the temperature range to 020K. Table 1 contains the numeric data taken from reference 1 used to create the various plots.

\section{Discussion/Conclusion}

The plots clearly show that at temperatures above 20 degrees Kelvin, pressure does not have a significant effect on the thermal conductivity of Helium. In figures 1 and 2 the four isobaric curves are indistinguishable. This is also evident from the numeric data in table 1. Comparing the thermal conductivity of any particular row in table 1 , the thermal conductivity does not change by more than one percent from one column to another.

Below 20K, the curves begin to diverge as they approach the saturation dome. This affect is more pronounced at pressures above the critical pressure of $0.23 \mathrm{MPa}$, At which point the fluid is neither a liquid or vapor. At pressures between atmospheric pressure and 0.01MPa, the 
thermal conductivity of Helium is independent of pressure below $20 \mathrm{~K}$ as well as above $20 \mathrm{~K}$.

\section{Reference}

1. "Thermophysical Properties of Helium-4", V. Arp and R. McCarty, November 1989. 


\section{Figure 1: He Thermal Conductivity vs. Temperature, 0-600K.}

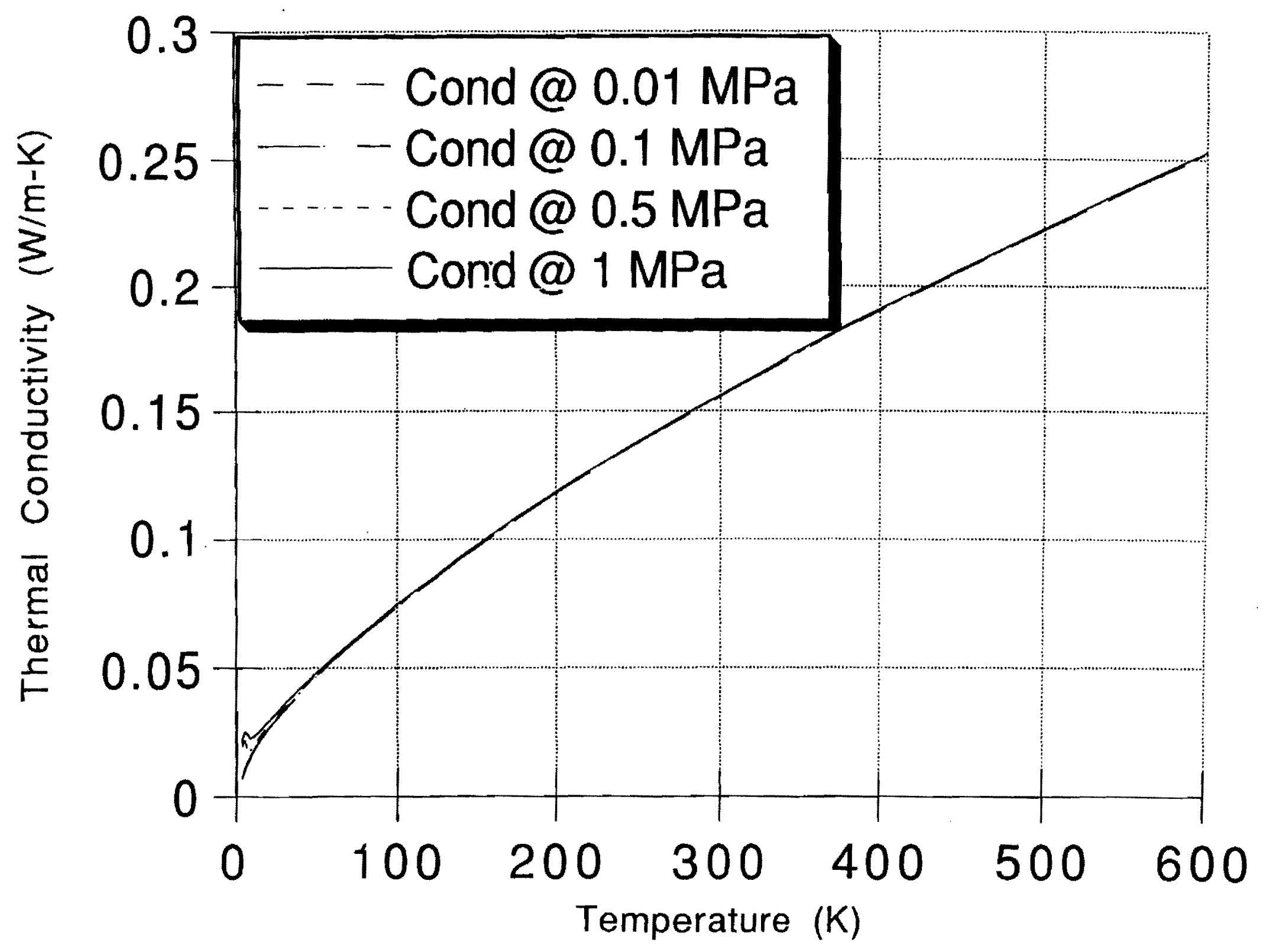


Figure 2: He Thermal Conductivity vs. Temperature, $0-300 \mathrm{~K}$.

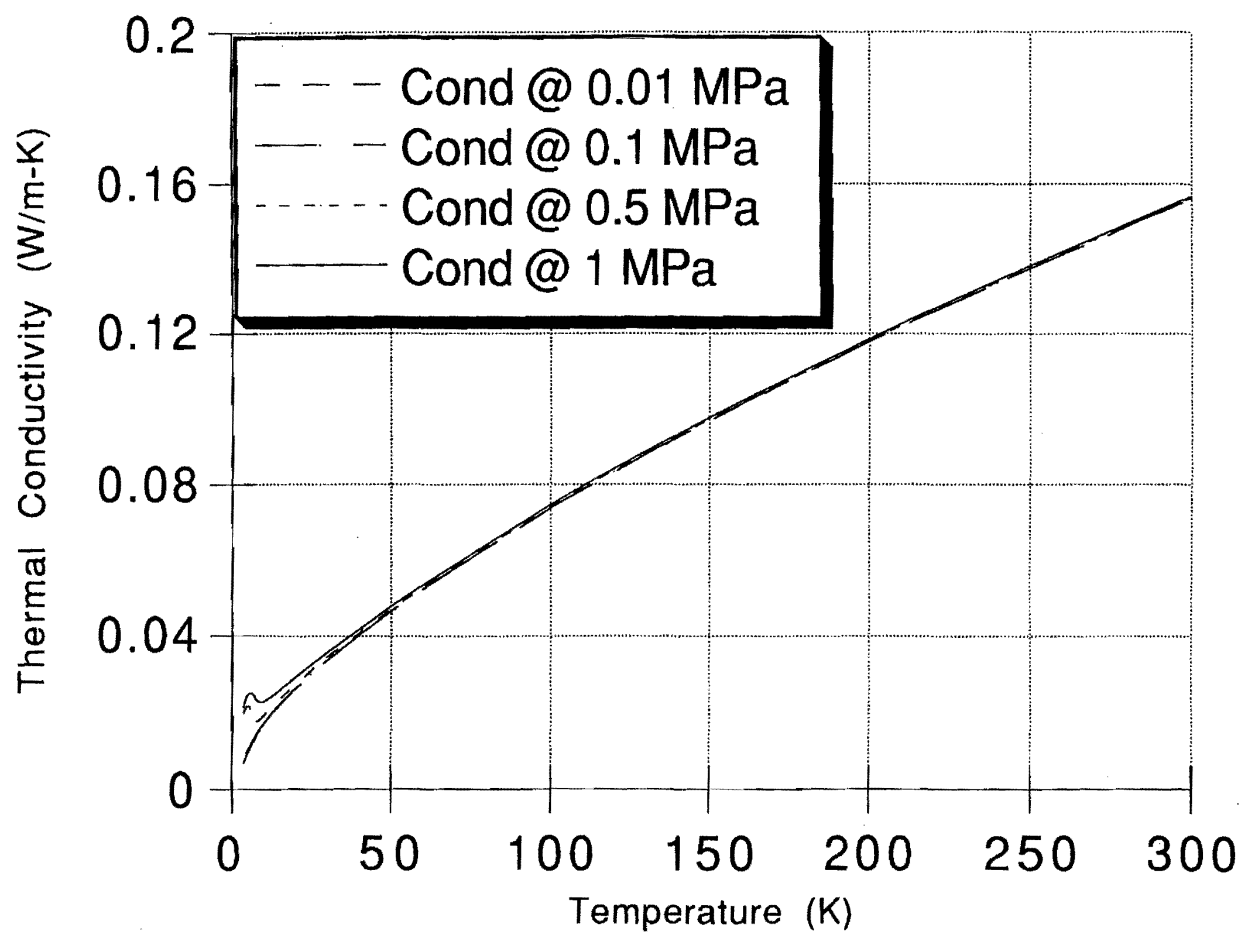


Figure 3: He Thermal Conductivity vs. Temperature, $0-20 \mathrm{~K}$.

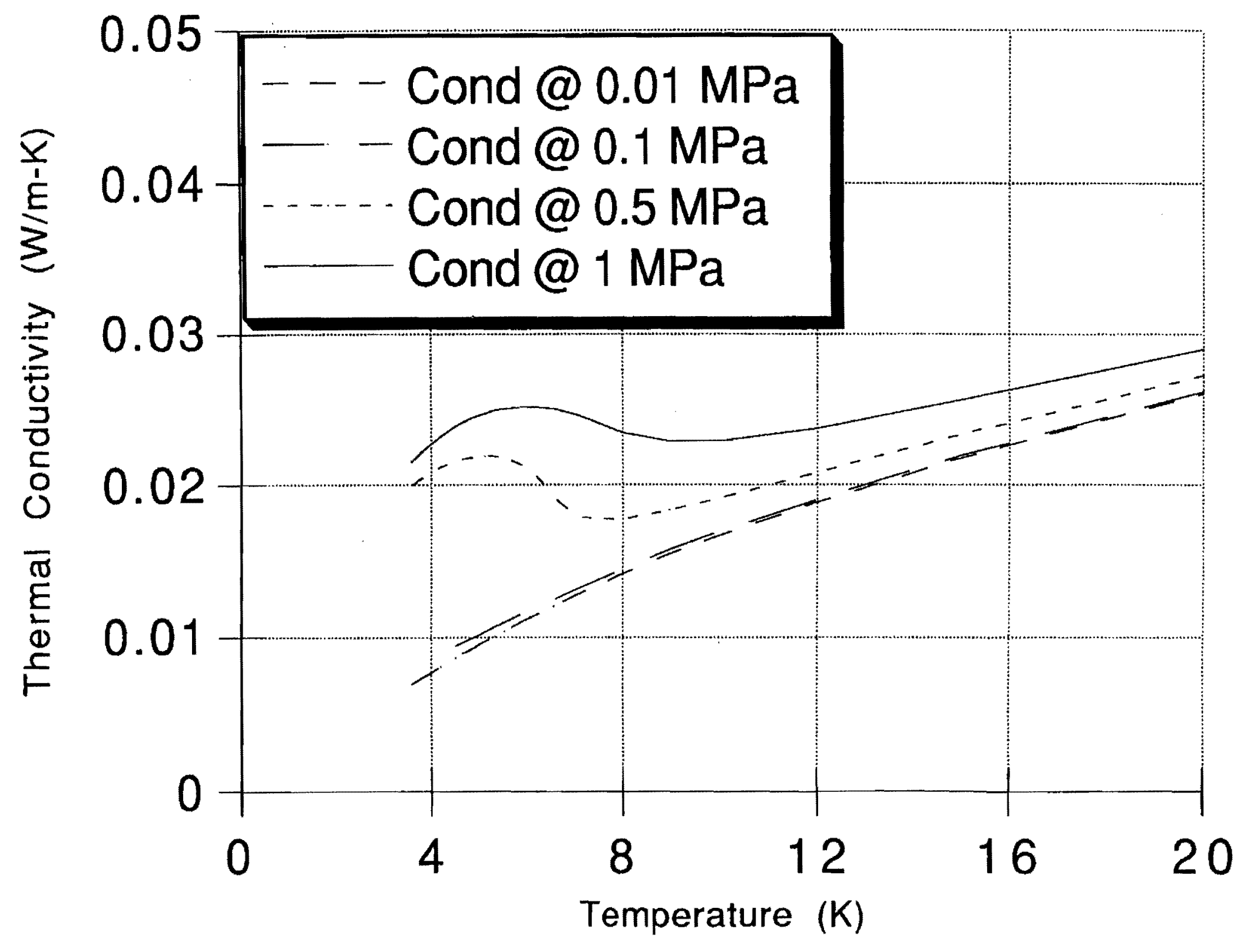


Table 1. Hellum Temperature and Thermal Conductlvity (W/m-K) Data

\begin{tabular}{|c|c|c|c|}
\hline Cond.@0.01 MPa & Cond.@0.1 MPa & Cond.@ $0.5 \mathrm{MPa}$ & Cond.@1 MPa \\
\hline 0.00696 & & 0.01994 & 0.02151 \\
\hline 0.00755 & & 0.02064 & 0.02242 \\
\hline 0.00812 & & 0.02118 & 0.0232 \\
\hline 0.00868 & 0.009449 & 0.02156 & 0.02385 \\
\hline 0.00923 & 0.009917 & 0.02179 & 0.02436 \\
\hline 0.00959 & 0.01023 & 0.02186 & 0.02463 \\
\hline 0.00977 & 0.01039 & 0.02187 & 0.02475 \\
\hline 0.01011 & 0.0107 & 0.02186 & 0.02494 \\
\hline 0.01045 & 0.011 & 0.02179 & 0.02507 \\
\hline 0.01127 & 0.01175 & 0.02114 & 0.02521 \\
\hline 0.01205 & 0.01247 & 0.01918 & 0.02507 \\
\hline 0.01279 & 0.01317 & 0.01787 & 0.02464 \\
\hline 0.01418 & 0.0145 & 0.01767 & 0.02346 \\
\hline 0.01546 & 0.01573 & 0.01831 & 0.02286 \\
\hline 0.01665 & 0.01689 & 0.01911 & 0.02289 \\
\hline 0.01883 & 0.01903 & 0.02081 & 0.02375 \\
\hline 0.02175 & 0.02191 & 0.02332 & 0.02563 \\
\hline 0.02608 & 0.0262 & 0.02726 & 0.02904 \\
\hline 0.02999 & 0.03009 & 0.03095 & 0.03242 \\
\hline 0.03362 & 0.03372 & 0.03445 & 0.03571 \\
\hline 0.04036 & 0.04045 & 0.04103 & 0.04201 \\
\hline 0.0466 & 0.04668 & 0.04718 & 0.048 \\
\hline 0.05248 & 0.05255 & 0.053 & 0.05372 \\
\hline 0.06344 & 0.06352 & 0.06391 & 0.0645 \\
\hline 0.07364 & 0.07371 & 0.07408 & 0.0746 \\
\hline 0.08326 & 0.08333 & 0.08368 & 0.08416 \\
\hline 0.09243 & 0.0925 & 0.09284 & 0.09329 \\
\hline 0.1012 & 0.1013 & 0.1016 & 0.1021 \\
\hline 0.1097 & 0.1098 & 0.1101 & 0.1105 \\
\hline 0.1179 & 0.118 & 0.1183 & 0.1187 \\
\hline 0.1259 & 0.126 & 0.1263 & 0.1267 \\
\hline 0.1337 & 0.1337 & 0.134 & 0.1344 \\
\hline 0.1412 & 0.1413 & 0.1416 & 0.142 \\
\hline 0.1486 & 0.1487 & 0.149 & 0.1494 \\
\hline 0.1559 & 0.156 & 0.1563 & 0.1566 \\
\hline 0.1735 & 0.1735 & 0.1738 & 0.1742 \\
\hline 0.1903 & 0.1904 & 0.1907 & 0.191 \\
\hline 0.2222 & 0.2223 & 0.2226 & 0.2229 \\
\hline 0.2523 & 0.2524 & 0.2527 & 0.25301 \\
\hline
\end{tabular}

